\title{
Serum Antibody Ig G and Ig M Titers for Opisthorchis felineus Correlate with Eggs in Faeces - a Comprehensive Study in Chuvash Republic, Russia
}

\author{
Vladimir U Emelianovi*, Tatiana Skvortsova1, Lilia V Mikhailova ${ }^{2}$, Elena N \\ Shamitova ${ }^{1}$
}

\begin{abstract}
The Cholangiocarcinoma is a. The risk of development of cholangiocarcinoma, generally a rare type of a liver tumor, increases during infection of Opisthorchiasis. For this reason the timely detection of Opisthorchiasis is important for Cholangiocarcinoma prevention. There are many studies which concern the detection of pathogenesis of Opisthorchis viverrini infection but a little known about Opisthorchis felineus. In this study we investigate a correlation of the eggs which are found in a faeces and are comparable with a serum Ig G and Ig $M$ antibody level that were detected with ELISA test in a large group of patients. The result is showing positive correlation between evidence of the Opisthorchis felineus eggs that were found in a faeces and antibody Ig $\mathbf{G}$ and Ig $\mathrm{M}$ level in a serum. Moreover the combination of two methods can improve the Opisthorchiasis diagnostic: the serum antibody and faeces investigation of eggs .
\end{abstract}

Keywords: The Opisthorchis felineus - eggs number - serum Ig G - Ig M - ELISA

Asian Pac J Cancer Prev, 17 (1), 281-283

\section{Introduction}

It was proved that the Opisthorchiasis infection was associated with the cholangiocarcinoma (Thanee et al., 2015). A helminth is one of the species of the opisthorchiasis infections that affect an hepatobiliary distal duct, a gall-bladder and a pancreatic duct. For this reason the timely detection of the Opisthorchiasis is important for preventing the cholangiocarcinoma and the prevention of spreading the infection. The disease of the Opisthorchiasis causes two main species: Opisthorchis viverrini with the main areas of distributions in Asia and Opisthorchis felineus (O.felineus) with the main geographical distribution in Russia and other European countries such as a Germany (Ogorodova et al., 2015). There are three main methods to diagnose this infection: a faeces investigation for eggs, an examination faeces with PCR method, and an immunologicalinvestigation of blood samples with ELISA (Rucksaken et al., 2015). PCR methods are very time consuming and expensive among all these methods. For this reason ELISA is still widely used as the best screening method.

The Chuvash republic is a region with a high density population (67.5 person per square kilometeres in 2015) but with low opisthorchiasis disease rate. One of the reasons is a low occurence of opisthorchiasis in Chuvashia as it is detected at the early stage of the disease, which may prevent spreading of the parasite, as it was shown in Thailand (Suwannahitatorn et al., 2013; Thaewnongiew et al., 2014). The main method of opisthorchiasis diagnostic in Chuvash republic is the ELISA test. In a literature there are several controversial papers which concern the correlation between anti Opisthorchis antibody level and number of eggs in faeces, but all this studies were investigated on Opisthorchis viverrini, but not on Opisthorchis felineus (Elkins et al., 1991). For this reason, we decided to explore the Opisthorchis felineus incidence rate in Chuvashia with a high density in population, but a low level of opisthorchiasis and to find a correlation between the eggs quantity in a faeces with the data from the laboratory ELISA test.

\section{Materials and Methods}

The total number of investigated patients was 25738 of adults with the age range between 18-86 years old. Patients took the test for helminthes eggs in 2012-2014 in the Hospital of the Chuvash republic. The eggs were diagnosed with Kato-Miura technique which is used for detection O. felineus infection. (Saengsawang et al., 2013). For the serological study for detection of the specific Ig $\mathrm{G}$ antibodies 4603 patients were investigated with ELISA for O. felineus specific Ig M and Ig G antibodies (Assay kit, ZAO Vector-Best, Novosibirsk, Russia).

${ }^{1}$ Department of Pharmacology, Clinical Pharmacology and Biochemistry, Medical School Chuvash State University, ${ }^{2}$ Department of Central Clinic \& Diagnostic Laboratory, Republican Clinical Hospital, Cheboksary, Chuvash Republic, Russia *For correspondence: vemelianov@mail.ru 
Vladimir U Emelianov et al

Table 1. Faeces Examination for Eggs

\begin{tabular}{cccc}
\hline Year & Men & Women & Men \& Women \\
\hline 2012 & 14 & 26 & 40 \\
2013 & 8 & 6 & 14 \\
2014 & 12 & 8 & 20 \\
Total & 34 & 40 & 74 \\
\hline
\end{tabular}

Table 2. The ELISA Test Results for Anti-Opisthorchis Antibodies

\begin{tabular}{lccc}
\hline Year & Men & Women & Men \& Women \\
\hline 2012 & 10 & 11 & 21 \\
2013 & 16 & 18 & 34 \\
2014 & 12 & 18 & 30 \\
Total & 38 & 47 & 85 \\
\hline
\end{tabular}

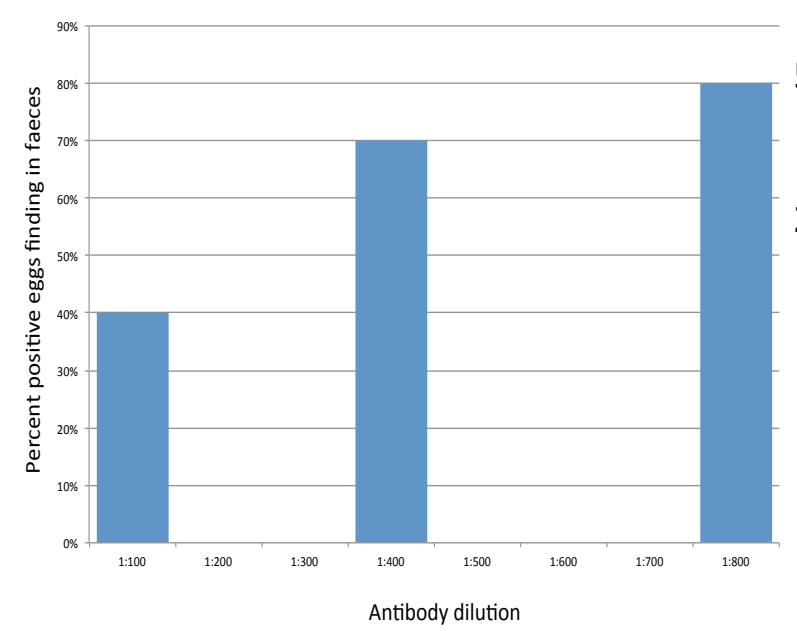

Figure 1. The Correlation between an Antibody Dilution Detected with ELISA and Intensity of the Infection. (Percent Positive Eggs finding in faeces), p- value $>0.05$

\section{Statistical analyses}

All groups of patients were assessed with the different estimates with using 1-way ANOVA test (Bonferroni multiple comparison test) and at p-value equal at less or 0.05 consider as significant.

\section{Results}

The total number of patients with $\mathrm{O}$. felineus eggs found in faeces shown in the table 1 . The Spreading of infection in adult patients was low and the total amount was 74 from 25738 examined patients. However, in 10 patients with positive ELISA, the eggs were detected after one or two times re-examination. ELISA was performed on 4603 patients with 85 positive results (Table 2).

In $10 \%$ seropositive patients Ig $\mathrm{G}$ the antibody level was 1:800, other seropositive patients had the antibody level from 1:100 to 1:400. 29 patients with the positive results for Ig G and Ig M (39\% from all the seropositive patients) after ELISA carried out a faeces examination for eggs. During examination O. felineus eggs in faeces were detected only in $40 \%$ of the cases in seropositive patients with Ig G and Ig M with 1:100 level, if patients had the quantity of 1:400-1:800 and the eggs were found in $70 \%$ and $80 \%$ correspondingly (Figure 1 ).

\section{Discussion}

In this study for the first time a positive correlation was shown between $\mathrm{O}$. felineus antibody level and eggs in faeces in a large number of patients. Before the comparable results were shown of the O. viverrini infection (Elkins et al., 1991; Watwiengkam et al., 2013). Also the combination of two methods was found: the faeces study for eggs with Kato-Miura technique and ELISA for Opisthorchis specific antigens, it had the direct correlation relationship which can improve $\mathrm{O}$. felineus diagnostic results (Starostina and Paniushkina, 2014). Levels of antigen in the serum significantly correlated with number of $\mathrm{O}$. felineus egg per gram. The faeces were shown in several studies but there are small numbers of patients that lead to controversial results with positive correlation or negative results (Akai et al., 1995; Watwiengkam et al., 2013).

These results can possibly show the similarity in pathogenesis and immune response between both species $\mathrm{O}$. viverrini and $\mathrm{O}$. felineus. The detection of the Opisthorchiasis by MAb-ELISA ELISA is sensitive and specific and there is more sensitivity than microscopic faeces examination for eggs (Sirisinha et al., 1995). There are not only serums but also a salivary IgG O. viverrini antigens that can be used for the opisthorchiasis infections detections (Chaiyarit et al., 2011). The Immune response for the $\mathrm{O}$. felineus is more associated with the somatic p105 and three egg an antigen proteins, such as p74, p70, p64 and less with excretory and secretory 20, 11, 25 antigens proteins (Chaiyarit et al., 2011). Animals were infected by the opisthorchis eggs production was coincided with the IgG appearance (Nockler et al., 2003). A strong immune response against the opisthorchiasis is associated by the increasing antigens as the parasite was maturing (Sripa and Kaewkes, 2000). An Immunological response to liver fluke antigens was also correlated with the severe liver ultrasonography findings, including the cholangiocarcinoma. (Akai et al., 1994). An increasing level of Ig G occurs when observed of hepatobiliary disease complication and when the cholangiocarcinoma is developing (Pinlaor et al., 2012).

The number of eggs in faecal consistency and the worm related with the burden in human, can be change to the during treatment, in particular the praziquantel treatment,in first few days number of eggs increased per gram feces and then it decreased to very low number in a several days . (Bychkov et al., 1990; Sithithaworn et al., 1991). In animals in the hamster eggs production of the Opisthorchiasis also positive correlate with the worm size (Flavell et al., 1983).

\section{References}

Akai PS, Pungpak S, Chaicumpa W, et al (1994). Serum antibody response to Opisthorchis viverrini antigen as a marker for opisthorchiasis-associated cholangiocarcinoma. Trans $R$ Soc Trop Med Hyg, 88, 471-4.

Akai PS, Pungpak S, Kitikoon V, et al (1995). Egg-negative residents of an Opisthorchiasis-endemic area possess high levels of anti-Opisthorchis antibodies. Adv Exp Med Biol, 
371, 983-6.

Bychkov VG, Ivanskikh VI, Molokova OA, et al (1990). [A comparison of the count of Opisthorchis in the body of the host and of the eggs eliminated with the feces]. Med Parazitol (Mosk), 14-6.

Chaiyarit P, Sithithaworn P, Thuwajit C, et al (2011). Detection of salivary antibodies to crude antigens of Opisthorchis viverrini in opisthorchiasis and cholangiocarcinoma patients. Clin Oral Investig, 15, 477-83.

Elkins DB, Sithithaworn P, Haswell-Elkins M, et al (1991). Opisthorchis viverrini: relationships between egg counts, worms recovered and antibody levels within an endemic community in northeast Thailand. Parasitology, 102, 283-8.

Flavell DJ, Flavell SU, Field GF (1983). Opisthorchis viverrini: the relationship between egg production, worm size and intensity of infection in the hamster. Trans $R$ Soc Trop Med Hyg, 77, 538-45.

Nockler K, Dell K, Schuster R, et al (2003). Indirect ELISA for the detection of antibodies against Opisthorchis felineus (Rivolta, 1884) and Metorchis bilis (Braun, 1790) in foxes. Vet Parasitol, 110, 207-15.

Ogorodova LM, Fedorova OS, Sripa B, et al (2015). Opisthorchiasis: an overlooked danger. PLoS Negl Trop Dis, 9, 3563.

Pinlaor P, Pongsamart P, Hongsrichan N, et al (2012). Specific serum $\operatorname{IgG}$, but not IgA, antibody against purified Opisthorchis viverrini antigen associated with hepatobiliary disease and cholangiocarcinoma. Parasitol Int, 61, 212-6.

Rucksaken R, Haonon O, Pinlaor P, et al (2015). Plasma IgG autoantibody against actin-related protein 3 in liver fluke Opisthorchis viverrini infection. Parasite Immunol, 37, 340-8.

Saengsawang P, Promthet S, Bradshaw P (2013). Infection with Opisthorchis viverrini and use of praziquantel among a working-age population in northeast Thailand. Asian Pac J Cancer Prev, 14, 2963-6.

Sirisinha S, Chawengkirttikul R, Haswell-Elkins MR, et al (1995). Evaluation of a monoclonal antibody-based enzyme linked immunosorbent assay for the diagnosis of Opisthorchis viverrini infection in an endemic area. Am J Trop Med Hyg, 52, 521-4.

Sithithaworn P, Tesana S, Pipitgool V, et al (1991). Relationship between faecal egg count and worm burden of Opisthorchis viverrini in human autopsy cases. Parasitology, 102, 277-81.

Sripa B, Kaewkes S (2000). Localisation of parasite antigens and inflammatory responses in experimental opisthorchiasis. Int J Parasitol, 30, 735-40.

Starostina O, Paniushkina, II (2014). [The comparative characteristic of methods of laboratory diagnostic of opisthorchiasis]. Klin Lab Diagn, 44-6.

Suwannahitatorn P, Klomjit S, Naaglor T, et al (2013). A follow-up study of Opisthorchis viverrini infection after the implementation of control program in a rural community, central Thailand. Parasit Vectors, 6, 188.

Thaewnongiew K, Singthong S, Kutchamart S, et al (2014). Prevalence and risk factors for Opisthorchis viverrini infections in upper Northeast Thailand. Asian Pac J Cancer Prev, 15, 6609-12.

Thanee M, Loilome W, Techasen A, et al (2015). Quantitative changes in tumor-associated M2 macrophages characterize cholangiocarcinoma and their association with metastasis. Asian Pac J Cancer Prev, 16, 3043-50.

Watwiengkam N, Sithithaworn J, Duenngai K, et al (2013). Improved performance and quantitative detection of coproantigens by a monoclonal antibody based ELISA to diagnose human opisthorchiasis. Acta Trop, 128, 659-65. 